\title{
Cartographie Des Zones À Risque D'incendies De Forêts À L'aide Du SIG Et La Télédétection Dans L'arrière-Pays Du Port Tanger Med
}

\author{
Abderrahim Maktite \\ Ali Faleh \\ Département de Géographie, Faculté des lettres Saïs Fès, Maroc, \\ Laboratoire de Recherches, d'Etudes Géographiques, \\ Aménagement et Cartographie (LEGAC)
}

Doi: 10.19044/esj.2017.v13n32p205 URL:http://dx.doi.org/10.19044/esj.2017.v13n32p205

\begin{abstract}
Moroccan natural environments, in recent decades, have undergone considerable degradation which is related primarily to the development of populations and the pressure they have exerted on natural resources. One aspect of this degradation is forest fire that has accelerated alarmingly. The study area belongs to the forest area of Tangier which covers an area of 42.000ha. The latter is known for its sensitivity to heat, especially because of the nature of the vegetation cover, weather conditions (frequent and strong wind), and high population density. The present work aims to prioritize the plot study area according to the degree of fire risk of forests using the model established by Dagorne Y. Duche in 1994. To achieve this goal, the application of GIS and Remote Sensing is required to develop a fire risk map in the hinterland of Tangier Mediterranean port.
\end{abstract}

Keywords: Fire, GIS, remote sensing, Tangier Med port

Résumé

Les milieux naturels marocains ont subi au cours des dernières décennies des dégradations importantes liées en premier lieu au développement des populations et à la pression qu'elles ont exercée sur les ressources naturelles. L'un des aspects de cette dégradation réside dans les incendies de forêts qui se sont accélérés d'une façon alarmante. La zone d'étude appartient au domaine forestier de la direction provinciale des eaux et forêts et de la lutte contre la désertification de Tanger qui s'étale sur une superficie de 42.000ha. Cette dernière est connue par sa sensibilité au feu notamment à cause de la nature du couvert végétal, des conditions climatiques (vent chergui fort et fréquent) et de la forte densité de la population. Le 
présent travail a pour objectif de hiérarchiser la zone d'étude en parcelle selon le degré de risque de feu de forêts à l'aide du modèle établit par Dagorne Y. Duche en 1994. Pour atteindre cet objectif, l'application du SIG et la télédétection sera mobilisée pour élaborer la carte du risque d'incendie dans l'arrière-pays du port Tanger Med.

Mots-clés: Incendies de forêts, SIG, Télédétection, Port Tanger Med

\section{Introduction}

Le Maroc perd chaque année 3000 hectares de forêts qui partent en fumée depuis 1960 avec une moyenne de 290 incendies par an (Huffpost Maroc/ Ibrahim Bayo, 2016).

Les principales causes de cette régression sont dues aux activités humaines (prélèvement de bois, surpâturage ...) et aux changements climatiques qui manifestent par des longues périodes de sécheresse et des vents violents et desséchants (chergui).

L'analyse de la situation des incendies de forêts au Maroc, fait ressortir qu'au cours des 50 dernières années (1960-2009), près de 12912 incendies ont endommagé 149292 ha de forêts, soit une moyenne de 2986ha par an, avec un maximum en 1983 (11289 ha) et un minimum en 2002 (593 ha). Cette superficie moyenne annuelle représente $0,05 \%$ de la surface totale boisée du pays (HCEFLCD, 2009).

Le nombre d'incendies et les superficies incendiées en forêts marocaines sont jugés relativement élevés, eu égard au taux de boisement très faible du pays (8\%), à l'aridité du climat et aux contraintes rendant extrêmement difficile la reconstitution des espaces boisés (reboisement régénération) (HCEFLCD, 2009).

Malgré la gestion durable de la forêt réalisée par l'Etat depuis 1998, (programme forestier national, cadre stratégique à l'horizon 2020) dont les objectifs étaient de protéger la biodiversité et l'environnement et lutter contre les incendies des forêts, le risque est toujours présent, et les préventions exécutées par des moyens traditionnels restent insuffisantes. Tandis qu'actuellement la prévention avec des moyens modernes comme le SIG et la Télédétection est le moyen pour concrétiser les risques d'incendie à travers la cartographie.

Le présent travail s'inscrit dans cette perspective, il vise à évaluer spécifiquement la sensibilité des secteurs face au risque d'incendie à travers un modèle qui intègre plusieurs paramètres et données spatiales, à l'aide du SIG et Télédétection. 
Présentation de la zone d'étude

La zone d'étude se situe en amont de l'axe du port méditerranée et oued Ksar Sghir (Figure 1). Elle appartient à la péninsule de Tanger, située au Nord-ouest du Maroc. Elle couvre une superficie de $193 \mathrm{~km}^{2}$ et comporte trois bassins (bassin oued R'mel, bassin oued Ksar Sghir et bassin oued Ghlala), limitée au nord par la mer Méditerranée, à l'est par la ville de Tétouan, à l'ouest par la ville de Tanger et au sud par la ville de l'Arache.

Sur le plan administratif, la zone d'étude dépend de la région de Tanger- Tetouan- Al Hoceima, Province de Tanger, Cercle Al Anjra.

Du point de vue géologique, la zone d'étude appartient au domaine des Flyschs de l'unité structurale de Beni Ider. Cette unité structurale constitue une bande qui ceinture vers l'extérieur la dorsale calcaire et la zone prédorsalienne, du Détroit de Gibraltar jusqu'à Al Hoceima. Les nappes de flyschs sont constituées de terrains d'âges allant du Jurassique inférieur à l'Oligocène (Michard, 1976).

Ce domaine considéré comme entièrement allochtone, est constitué essentiellement de turbidites dont la nature est sensiblement différente (plus argileuse) que celle des autres domaines, Selon (Durand Delga et al., 1960).

Le faciès lithologique prédominant au niveau de secteur de travail est constitué de marne calcaires rouge suivies de marnes gréseuses micacées appartenant au flysch de Beni-Ider au niveau de la commune Ksar Majaz au nord-est, l'argile et les marnes d'âge Crétacé supérieur à Ksar Sghir au nordouest et le calcaires marneux argileux et de flyschs de marnes sableuses micacées à la commune Tghramt au sud (TMSA, 2008).

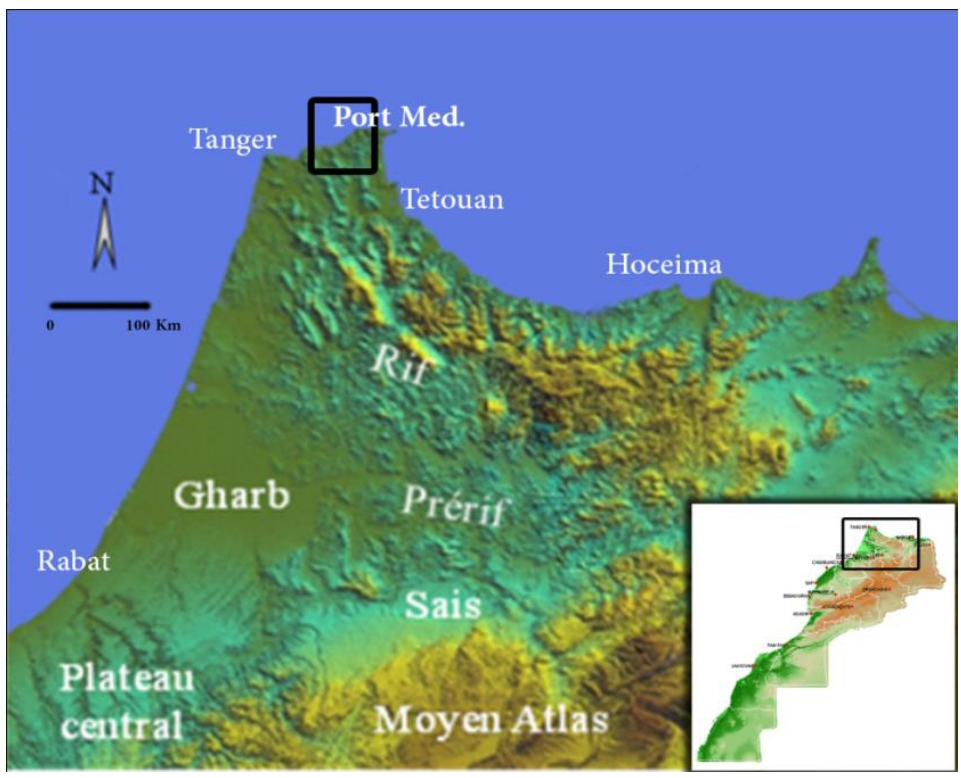

Figure 1. Croquis de la zone 
Le climat de la région est de type subhumide à saison hivernale régulièrement pluvieuse et fraîche, et à saison estivale sèche et chaude. La région reçoit entre $600 \mathrm{~mm} / \mathrm{an}$ et $800 \mathrm{~mm} / \mathrm{an}$.

Le régime pluviométrique est caractérisé par des pluies torrentielles et irrégulières. Les précipitations sont concentrées en hiver (entre décembre et février) tandis que la saison sèche coïncide avec la saison chaude estivale (entre juillet et août).

Historique des incendies dans les forêts de Tanger

Malgré que le nombre d'incendies de forêts enregistrés en 2015 soit supérieur à celui de l'année 2014, la superficie incendiée a diminuée de 63\%.

Tableau 1. Situations des incendies de forêts dans la province de Tanger

\begin{tabular}{|c|c|c|c|c|c|c|c|}
\hline Année & 2009 & 2010 & 2011 & 2012 & 2013 & 2014 & 2015 \\
\hline Nombre d'incendies & 52 & 52 & 63 & 70 & 46 & 28 & 53 \\
\hline Superficie incendiée & 42 & 39 & 61 & 950 & 86 & 121 & 45,97 \\
\hline
\end{tabular}

Source: DPEFLCD de Tanger) (

D'après le tableau le nombre d'incendies varie chaque année entre 30 et 70 avec une superficie incendiée entre 40 et 120 ha à l'exception de l'année 2012 qui a connu une grande dégradation des forêts avec une superficie de 950 ha (DPEFLCD, 2015).

- Situation des incendies de forêts dans la zone d'étude en 2015 Dans la zone d'étude, l'année 2015 a connu 43 incendies, les feux ont attaqué 39 ha.

Tableau 2. Espaces incendiés par commune dans la zone d'étude

\begin{tabular}{|c|c|c|}
\hline Commune Rurale & Nombre d'incendies & Superficie ravagée \\
\hline Ksar Al majaz & 6 & 10,78 \\
\hline ksar El Sghir & 1 & 0,2 \\
\hline Malloussa & 6 & 8,92 \\
\hline Tlata Taghramt & 4 & 10,09 \\
\hline CU de Tanger & 26 & 9,63 \\
\hline Totaux & 43 & 39,62 \\
\hline
\end{tabular}

Source: DPEFLCD de Tanger) (

Le Tableau 2 montre que les forêts les plus touchées par les incendies sont celles des communes Ksar Al majaz (10,78 ha), Tlata Taghramt (10,09 ha) et CU de Tanger avec une superficie de 9,63 ha (DPEFLCD, 2015).

Approche methodologique

L'évaluation des risques d'incendies de forêt a fait l'objet de plusieurs recherches. Par ailleurs, plusieurs indices de risque de feu ont été établis. 
L'indice qui nous intéresse dans ce travail est conçu par Dagorne Y. Duche pour les forêts de la Méditerranée.

Ce choix, en aucun cas aléatoire est peut être justifié par deux raisons: la première c'est l'appartenance du Maroc aux pays Méditerranéens, la seconde la disponibilité des données sur le terrain pour établir et confirmer le modèle.

L'indice du modèle est donné par la formule suivante: $\mathrm{IR}=5 \mathrm{IC}+2 \mathrm{IH}+\mathrm{IM}$

d'où:

IR: Indice de risque de feu.

IC: Indice de combustibilité.

IH: Indice d'occupation humaine.

IM: indice Topomorphologique.

Indice Topomorphologique (IM)

La pente a une influence considérable sur la vitesse de propagation du feu; le rayonnement utile à la propagation du feu est important. En effet, pour une montée de feu, les forces de poussée exercées verticalement à un angle par la direction de propagation du feu, plus fermé que la pente sont raides. Donc on peut observer le flux de gaz chauds du feu vers la végétation encore imbrulée (Guettouche et al., 2011).

Trois paramètres morphologiques impliqués dans le modèle topomorphologique: la pente, l'exposition et l'altitude. La combinaison de ces trois paramètres est exprimée par l'équation suivante:

$\mathrm{IM}=3 \mathrm{p}+(\mathrm{m}+\mathrm{e})$
p: la pente
m: la topomorphologie
e: l'exposition

Indice de Combustibilité (IC)

Les paramètres reflétant la sensibilité de la végétation au feu : le type des arbres et ses structures. Ceci est également un facteur important dans la propagation des incendies.

Pour estimer la combustibilité, on adopte le modèle développé par (Mariel, 1995) dont L'indice de combustibilité est sous forme:

$\mathrm{IC}=39+0,23 \mathrm{BV}(\mathrm{E} 1+\mathrm{E} 2-7,18)$

BV: le biovolume de la végétation.

E1: les notes de combustibilité pour les ligneux hauts.

herbacées.

E2: les notes de combustibilité pour les ligneux bas ou les 
L'indice d'occupation Humaine (IH)

Dans la plupart des cas, les causes des incendies liés à l'homme restent inconnues, principalement pour éviter délibérément l'attribution de la responsabilité pour les dommages et l'indemnisation des coûts des dommages.

Puisque l'homme est responsable de la plupart des mises à feu (volontaires et involontaires) des forêts, il est impossible de modéliser le comportement humain. L'approche statistique développée par J.G.Robin en 2006 montre une corrélation claire entre le nombre des foyers près d'une route ou d'habitations.

Pour évaluer l'effet de l'influence humaine sur les risques d'incendie de forêts, on adopte le modèle suivant:

$\mathrm{IH}=\mathrm{IV}+2 \mathrm{ID}$

$$
\begin{aligned}
& \text { D'où: } \quad \text { IV : l'indice de voisinage. } \\
& \text { ID : L'indice de présence humaine. }
\end{aligned}
$$

Résultats et Discussions

L'indice de risque de feu, premier objectif de cette étude est conçu comme un modèle attribuant à chaque paramètre un coefficient de pondération en fonction de son influence sur la propagation du feu.

Indice de Topomorphologie (IM)

A partir du modèle numérique du terrain qui a été réalisé à partir de la carte topographique avec une échelle de $1 / 25000$, on a dérivé la carte des pentes, d'exposition des versants et l'altitude.

Ces trois paramètres topographiques interviennent dans la méthode afin de réaliser la carte d'indice topomorphologique.

La Carte des Pentes

La pente joue un rôle très important à la propagation du feu, autant la pente est forte autant le feu se déplace rapidement.

Tableau 3. Classes de pentes

\begin{tabular}{|c|c|c|c|}
\hline Classes de Pentes & Superficie $\left(\mathrm{Km}^{2}\right)$ & Pourcentage & code \\
\hline $\mathrm{P}<15 \%$ & 138,28 & $71,5 \%$ & 1 \\
\hline $15 \% \leq \mathrm{P}<30 \%$ & 53,28 & $27,61 \%$ & 2 \\
\hline $30 \% \leq \mathrm{P}<60 \%$ & 1,8 & $0,83 \%$ & 3 \\
\hline $\mathrm{P} \geq 60 \%$ & 0,32 & $0,06 \%$ & 4 \\
\hline
\end{tabular}




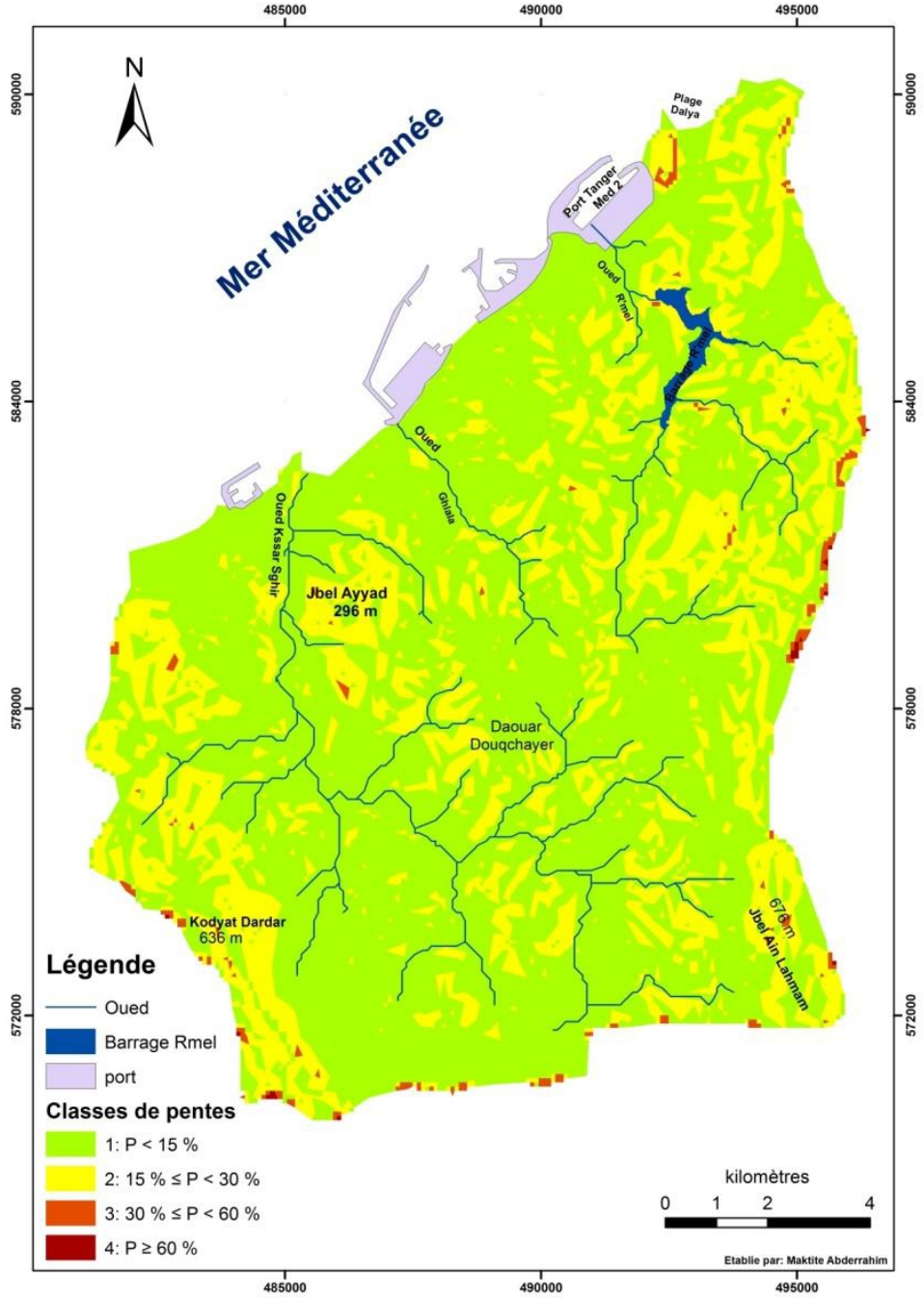

Figure 2. Carte de pentes

La carte des classes de pente (Figure 2) renseigne sur la variation de la raideur des pentes, cette variation joue un rôle très important car elle peut influencer le degré de propagation de feu (Saidi, 1994).

Cette dernière montre que la pente dans la plupart des terrains est faible à modérée, cependant, les pentes extrêmes ont une faible représentation sur la carte à la limite de la zone d'étude. 


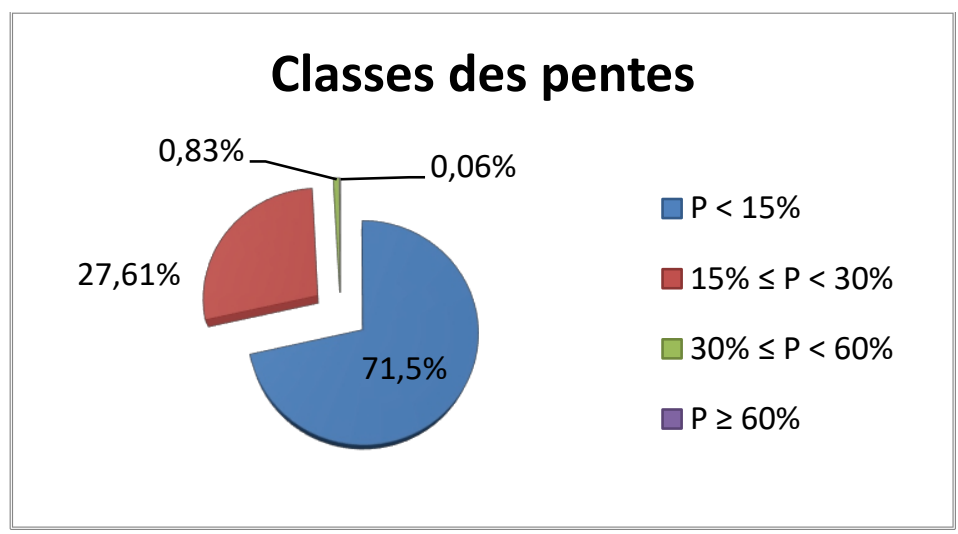

Figure 3. Répartitions des classes des pentes

La répartition des surfaces des différentes classes de pentes montre une prédominance de la classe 1 (pente de $<15 \%$ ) suivie par la classe 2 (pente 15-30); avec un décroissement à partir de la classe2 vers les classes de pentes les plus fortes; cela veut dire que la zone d'étude est généralement à des faibles à moyenne pente.

\section{La Carte d'exposition des Versants}

L'exposition détermine les versants chauds ou frais à partir de son ensoleillement, pour cela l'exposition des versants joue un rôle important à la propagation des inflammations.

Tableau 4. Classes d'expositions

\begin{tabular}{|c|c|c|c|}
\hline Orientation & Superficie $\left(\mathrm{Km}^{2}\right)$ & Pourcentage & code \\
\hline EST & 45 & $23,31 \%$ & 3 \\
\hline SUD & 33 & $17,11 \%$ & 2 \\
\hline OUEST & 63 & $32,64 \%$ & 1 \\
\hline NORD & 52 & $26,94 \%$ & 0 \\
\cline { 2 - 4 } & 193 & $100 \%$ & \multicolumn{2}{|c}{} \\
\cline { 2 - 4 } & \multicolumn{2}{|l}{}
\end{tabular}

\section{Exposition des versants}

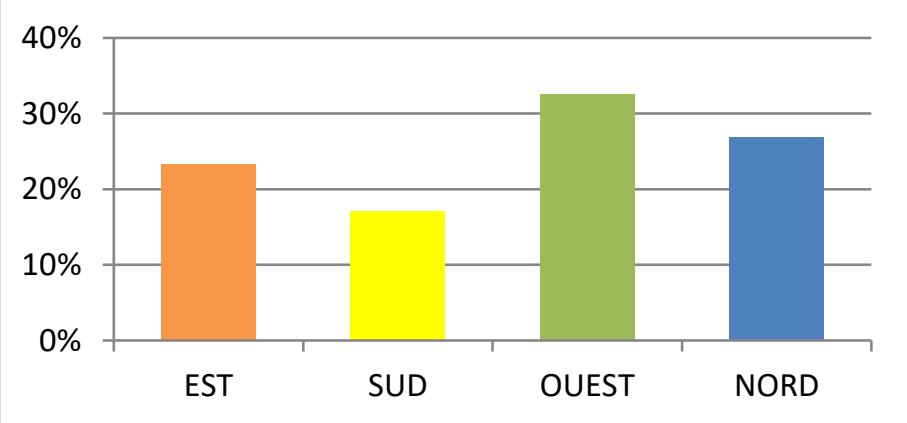

Figure 4. Répartitions des classes d'exposition des versants 
Normalement, les versants qui peuvent être menacés par les incendies sont exposés vers le SUD et l'EST car ils présentent les conditions les plus favorables pour une inflammation comme la dégradation des sols et le vent violant (chergui). Ces derniers représentent la moitié de superficie de la zone d'étude avec $55 \%$, ce qui rend ce facteur important, et de ce fait il nous incombe de le mettre en considération.

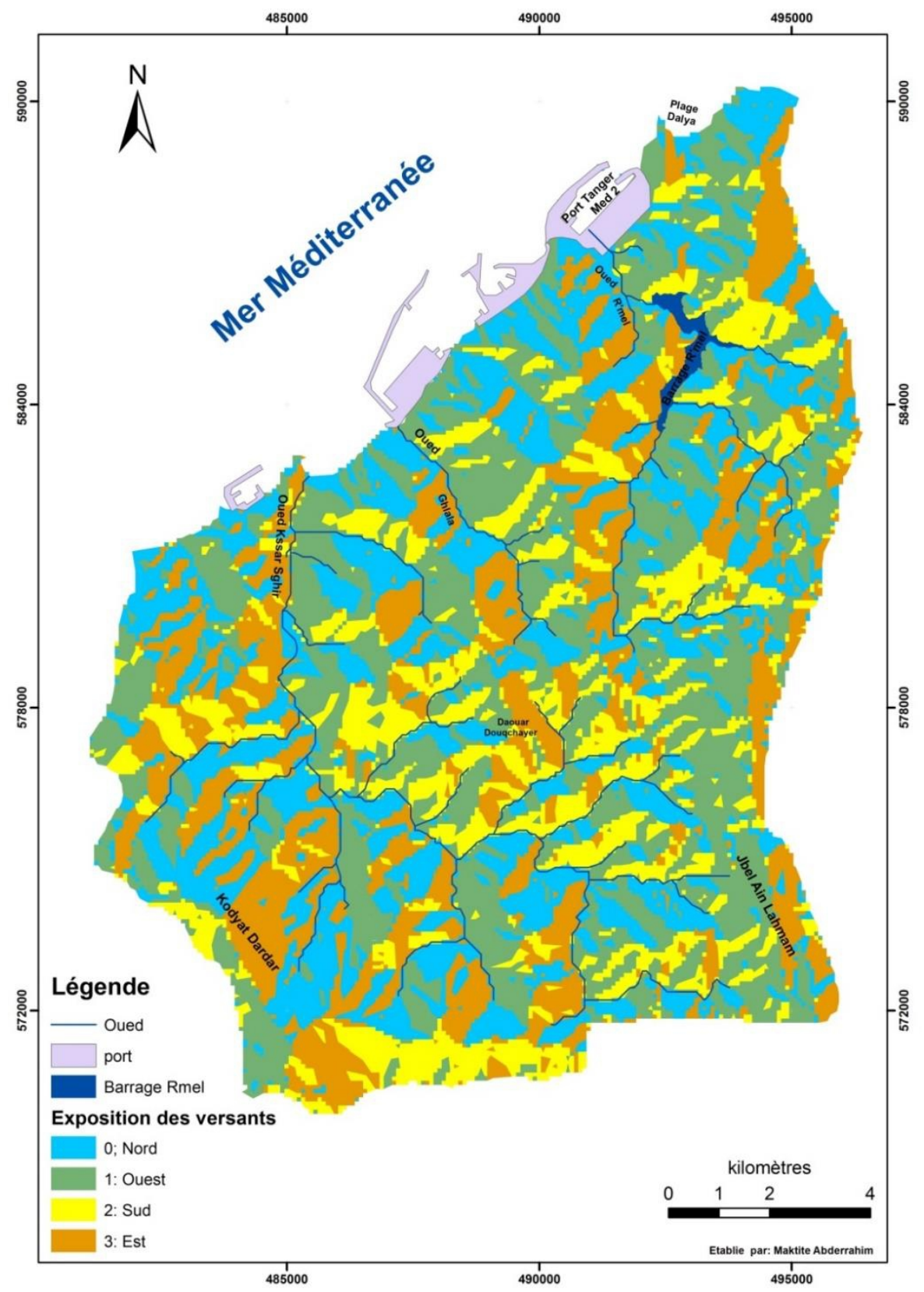

Figure 5. Carte d'exposition des versants 


\section{La Carte Topomorphologie}

La carte topomorphologique a été réalisée à partir du modèle numérique du terrain MNT. Elle est classée en quatre degrés d'altitude selon le Tableau 5:

Tableau 5. Classes topomorphologiques

\begin{tabular}{|c|c|c|c|c|}
\hline Classes des Pentes & Topographie & superficie $\left(\mathrm{Km}^{2}\right)$ & Pourcentage & code \\
\hline $\mathrm{P}<3 \%$ & Plaine & 8,5 & $4,23 \%$ & 1 \\
\hline $3 \% \leq \mathrm{P}<12,5 \%$ & Bas piémont & 96 & $49,74 \%$ & 2 \\
\hline $12,5 \% \leq \mathrm{P}<25 \%$ & Haut piémont & 84,38 & $43,62 \%$ & 3 \\
\hline $\mathrm{P} \geq 25 \%$ & Montagne & 4,8 & $2,41 \%$ & 4 \\
\cline { 3 - 5 } & & 193,68 & $100 \%$ & \\
\end{tabular}

Le feu agit différemment sur un terrain selon qu'il est plat ou selon qu'il présente des aspérités très marquées.

Dans la zone d'étude, la majorité des terrains se trouvent dans les bas ou les hauts piémonts avec un pourcentage de 49,74\% de la surface pour les bas piémonts et $43,62 \%$ pour les hauts piémonts, les zones de plaines et les hautes montagnes ne présentent que $4,2 \%$ pour les plaines et $2,4 \%$ pour les hautes montagnes.

La carte de l'indice topomorphologique (Figure 6) est élaborée par la superposition de la carte des pentes, la carte d'exposition et la carte topomorphologique selon la formule d'indice topomorphologique.

La combinaison entre les trois cartes donne une idée sur l'influence du relief sur la propagation des incendies.

Tableau 6. Classes d'indice de topomorphologique

\begin{tabular}{|c|c|c|c|c|}
\hline IM & Signification & Superficies $\left(\mathrm{km}^{2}\right)$ & Pourcentage\% & Code \\
\hline IM $<9$ & Peu favorable & 118,1 & $61,21 \%$ & 1 \\
\hline $9 \leq \mathrm{IM}<14$ & Moyennement favorable & 59,3 & $30,69 \%$ & 2 \\
\hline $14 \leq \mathrm{IM}<19$ & Favorable & 15 & $7,74 \%$ & 3 \\
\hline IM $\geq 19$ & Très favorable & 1,28 & $0,36 \%$ & 4 \\
\cline { 3 - 5 } & & 193,68 & $100 \%$ & \multicolumn{2}{c}{} \\
\cline { 3 - 5 } & & \multicolumn{3}{c}{}
\end{tabular}

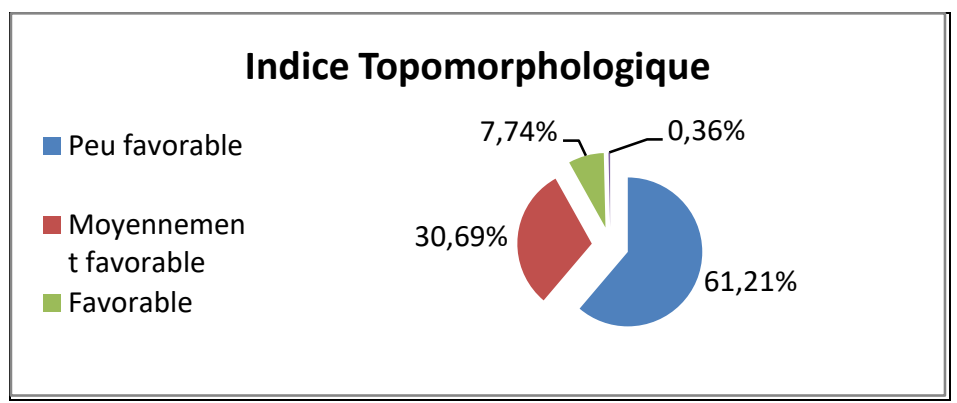

Figure 6. Répartition de l'indice topomorphologique 
La base de données de la carte de l'indice topomorphologique montre que $61 \%$ de la surface ont des conditions peu favorables à l'accentuation de feu, suivie par les conditions moyennement favorables avec 30\%, par contre les zones favorables et très favorables ne représentent que $8 \%$.

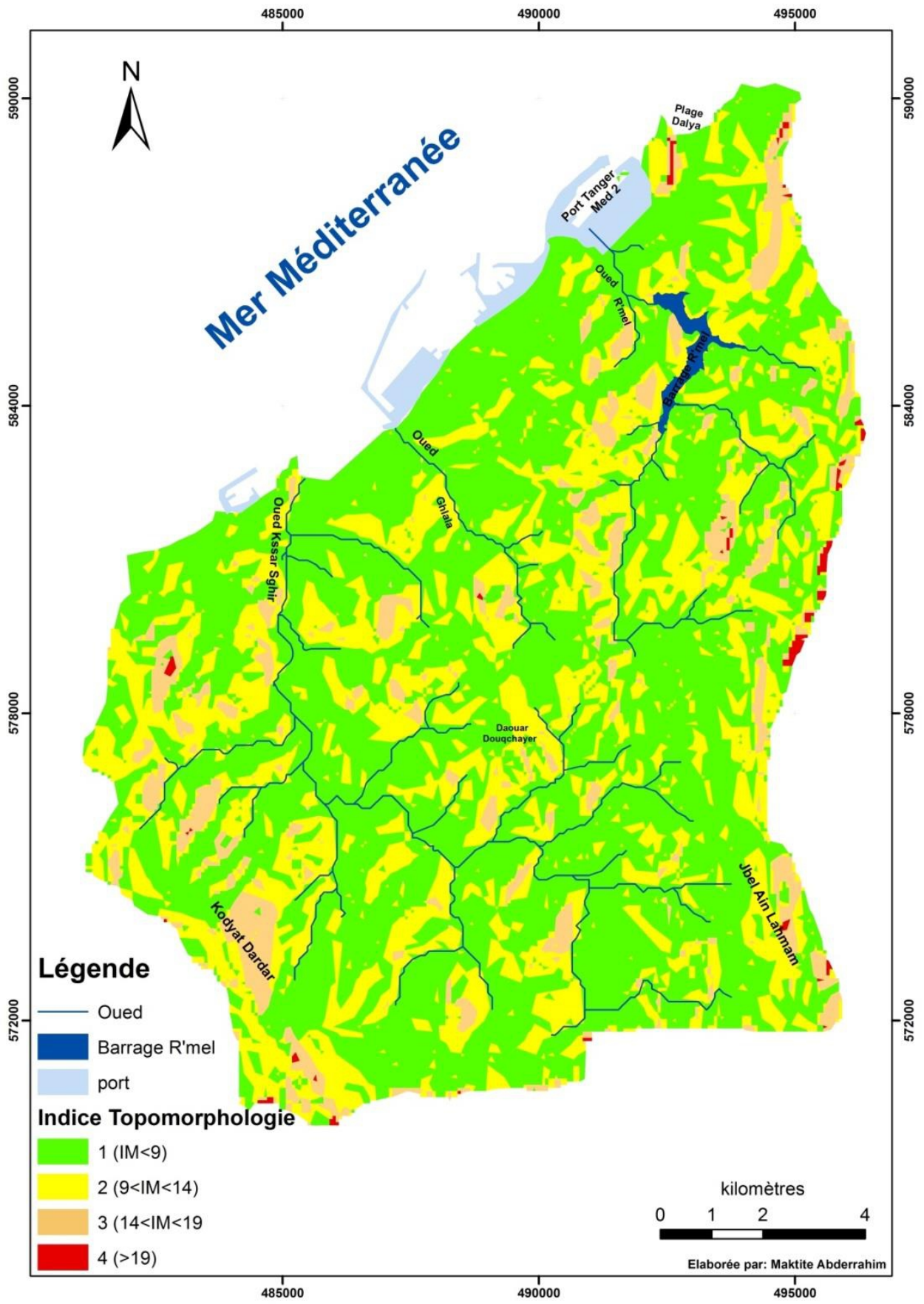

Figure 7. Carte de l'indice topomorphologique 
La répartition des classes d'indice topomorphologique (Figure 6) montre une dominance des classes (peu favorables) et (moyennement favorables) qui se trouvent partout dans la zone, pour la classe (favorables), elle est sur quelques terrains dans le sud-ouest, le nord-ouest et le nord-est, cependant, les terrains très favorables aux incendies se caractérisent par leur rareté. La base des données de la carte de l'indice topomorphologique montre que $61 \%$ de la surface ont des conditions peu favorables à l'accentuation de feu, suivi par les conditions moyennement favorables avec 30\%, par contre les zones favorables et très favorables ne représentent que $8 \%$.

\section{L'indice de Combustibilité}

Le modèle de dagorne contient des indices pour déterminer la teneur en eau du combustible à partir des caractéristiques biologiques des espèces et de la biomasse végétale, pour prédire la distance maximum à toute source de mise à feu, et estimer la probabilité d'éclosion d'un feu compte tenu des conditions du milieu.

\section{Carte de l'indice de Combustibilité}

La carte de combustibilité a été réalisée à partir de la carte NDVI (Figure 8) du plan d'aménagement des eaux et forêts de Tanger (2004). La densité de couvert végétale présentée par la structure et la combustibilité (BV: la biomasse des couverts végétales), en plus son inflammabilité (le pourcentage de teneur en eau) sont deux facteurs importants à l'extension des incendies des forêts. Ces deux facteurs ont été calculés à partir de la base de donnée de la carte de végétation en suivant la formule de l'indice de combustibilité; qui a été déterminée à partir des images satellitaires et les notes d'intensité calorique des espèces (E1 et E2) ont été extraites de celle établie par la (CEMAGREF). 


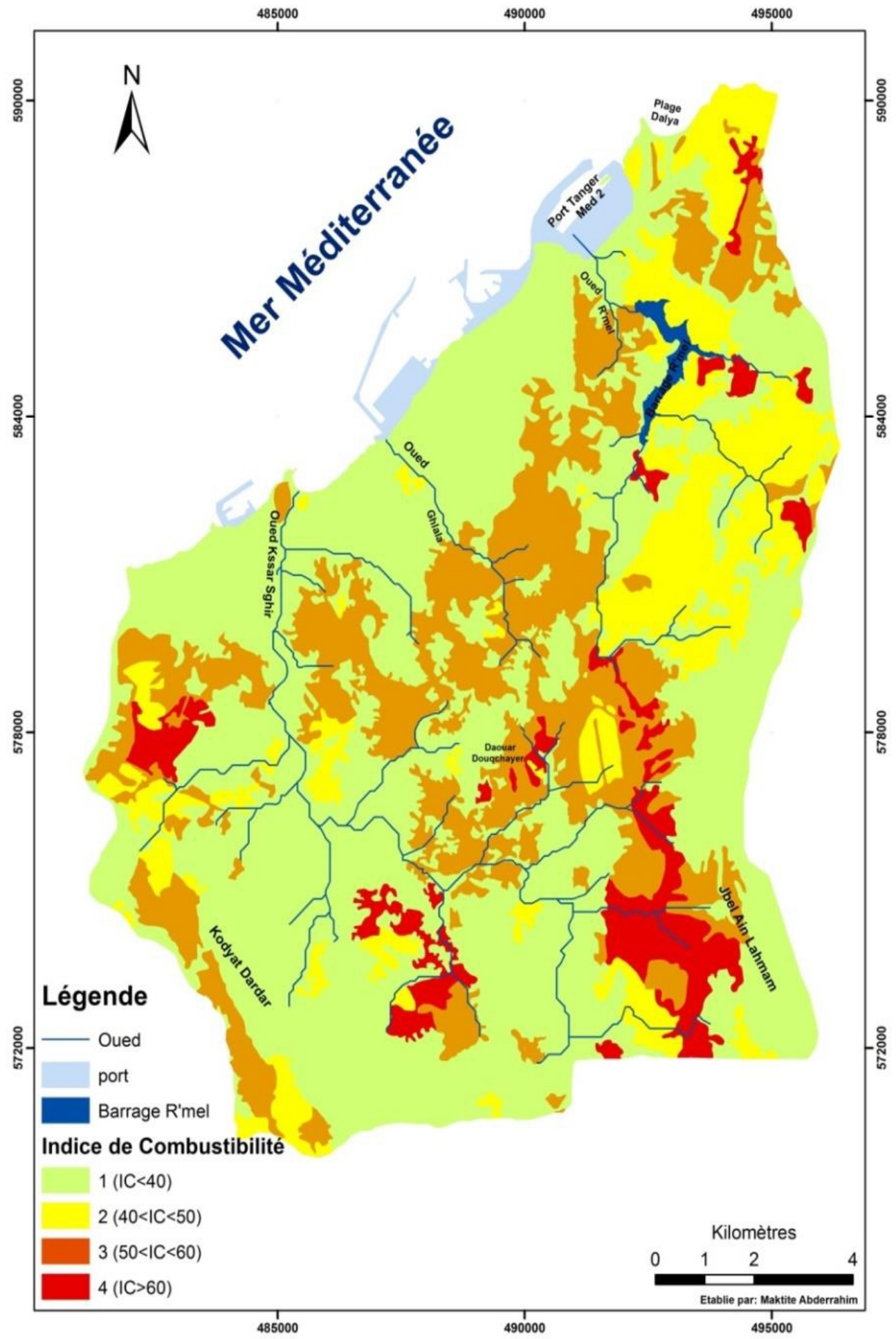

Figure 8. Carte de l'indice de combustibilité 
La carte de l'indice de combustibilité a été classée en 4 classes selon la résistance en feu; La répartition des différents types d'occupation des sols (Figure 9) répond à l'influence conjuguée d'une multitude de paramètres écologiques (le climat, le type de sols, l'altitude, l'exposition des versants, la pente, etc.) et des actions anthropiques (prélèvements de bois, etc..).

Sur les zones de haute altitude, et notamment dans le Nord-Ouest, Sud-Est et au milieu, on remarque la présence des superficies importantes des forêts et des arbustes denses, cela peut s'expliquer par la quantité d'eau que reçoivent ces parties et leur exposition vers le Nord et l'Est.

On note aussi la présence de quelques terrains nus et les cultures sèches au milieu de la zone à travers l'écoulement d'oued Ksser Sghir (du Sud vers le Nord).

À partir de cette répartition on peut dire que les zones de haute altitude sont favorables voire très favorables au risque d'incendies par la présence de type des arbres avec une intensité calorique élevée $(E 1=7)$.

Le traitement de la base de données a permis la réalisation du tableau, dans lequel figure les pourcentages des superficies des différentes classes d'indice de combustibilité.

Les résultats obtenus de la carte montrent que $57 \%$ de la surface forestière ont de faible risque à feu avec une superficie de $110,6 \mathrm{~km}^{2}$ suivis par les zones à risque élevé avec $22 \%$, par contre les terrains à risque modéré et très élevé présentent $14 \%$ et $6 \%$.

Tableau 7. Classes d'indice de combustibilité

\begin{tabular}{|c|c|c|c|c|}
\hline Classe d'IC & Note de Risque & Superficie en $\mathrm{km}^{2}$ & Pourcentage & Code \\
\hline IC $<40$ & FAIBE & 110,6 & $57,10 \%$ & 1 \\
\hline $40 \leq \mathrm{IC}<50$ & MODERE & 28,29 & $14,61 \%$ & 2 \\
\hline $50 \leq \mathrm{IC}<60$ & ELEVE & 43,19 & $22,29 \%$ & 3 \\
\hline IC $\geq 60$ & TRES ELEVE & 11,6 & $5,99 \%$ & 4 \\
\cline { 3 - 5 } & & 193,68 & $100,00 \%$ & \multicolumn{2}{c}{} \\
\cline { 3 - 5 } & &
\end{tabular}

Figure 9. Histogramme d'indice de combustibilité

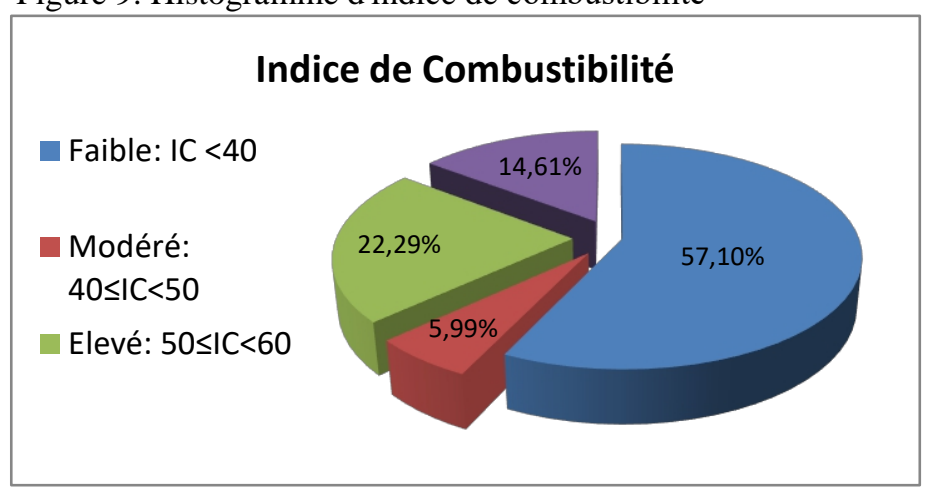




\section{L'indice d'occupation Humaine}

Pour évaluer l'effet humain sur les risques d'incendie, on a digitalisé les différentes routes, chemins et les zones d'habitats à partir de Google Earth avec une zone d'influence de $100 \mathrm{~m}$ autour de chaque élément et enfin, on a codé ces derniers de 1 à 4 selon l'éloignement des forêts.

Figure 10. Carte des classes de risque liée à l'habitat Figure 11. Carte des classes de risque liée aux réseau routière
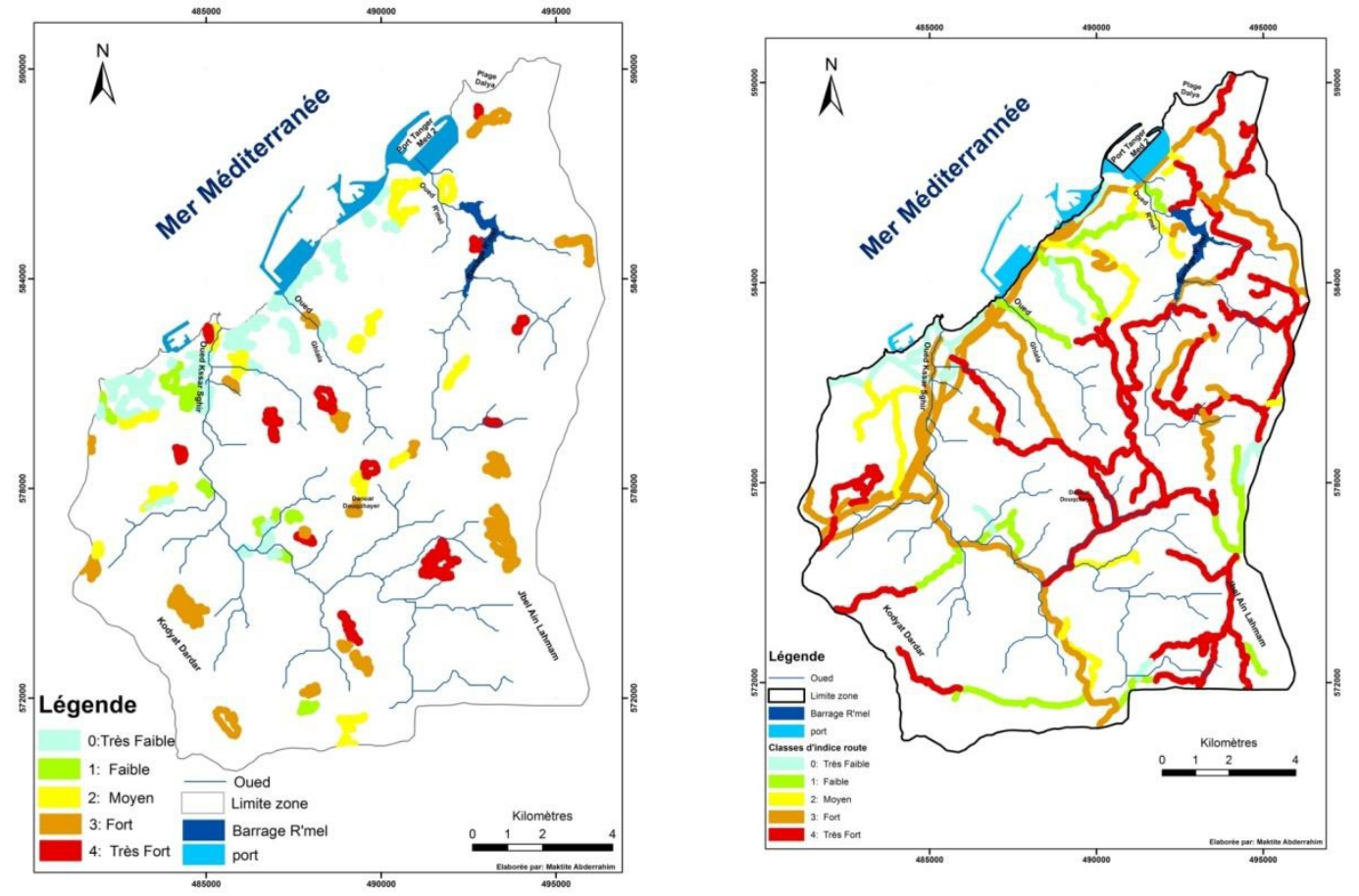

Carte de l'indice de Risque de Feu

Trois couches sont superposées pour la réalisation de l'indice de risque de feu, tels que l'indice topomorphologique, l'indice de combustibilité et l'indice humaine selon la formule suivante:

$\mathrm{IR}=5 \mathrm{IC}+2 \mathrm{IH}+\mathrm{IM}$

Tableau 8. Classes d'indice de risque de feu

\begin{tabular}{|c|c|c|c|c|}
\hline \multirow{2}{*}{ Classes d'IR } & Signification & Superficies & Pourcentage & CODE \\
\hline IR $<6$ & Risque Faible & 1,43 & $1 \%$ & 1 \\
\hline $6 \leq \mathrm{IR}<9$ & Risque Moyenne & 80,53 & $42 \%$ & 2 \\
\hline $9 \leq \mathrm{IR}<12$ & Risque Fort & 17,3 & $9 \%$ & 3 \\
\hline IR $\geq 12$ & Risque Très Fort & 94,42 & $49 \%$ & 4 \\
\cline { 2 - 4 } & & 193,68 & $100 \%$ & \multicolumn{2}{c}{} \\
\cline { 3 - 4 }
\end{tabular}


Le Tableau 9 montre que les zones à risque très fort et moyen sont les plus dominantes avec un pourcentage respectivement de $49 \%$ et $42 \%$. Pour les autres classes de risques faibles et forts, ils sont très faibles par rapport aux autres classes.

Les résultats du tableau montrent que la zone comporte des surfaces favorables à très favorables pour le déclenchement des incendies et cela émane, à coup sûr des peuplements ou des espèces végétales. S'ajoutent à ces causes la présence des activités commerciales et économiques exercés dans la région comme le port, les carrières et le tourisme qui accélèrent ce phénomène.

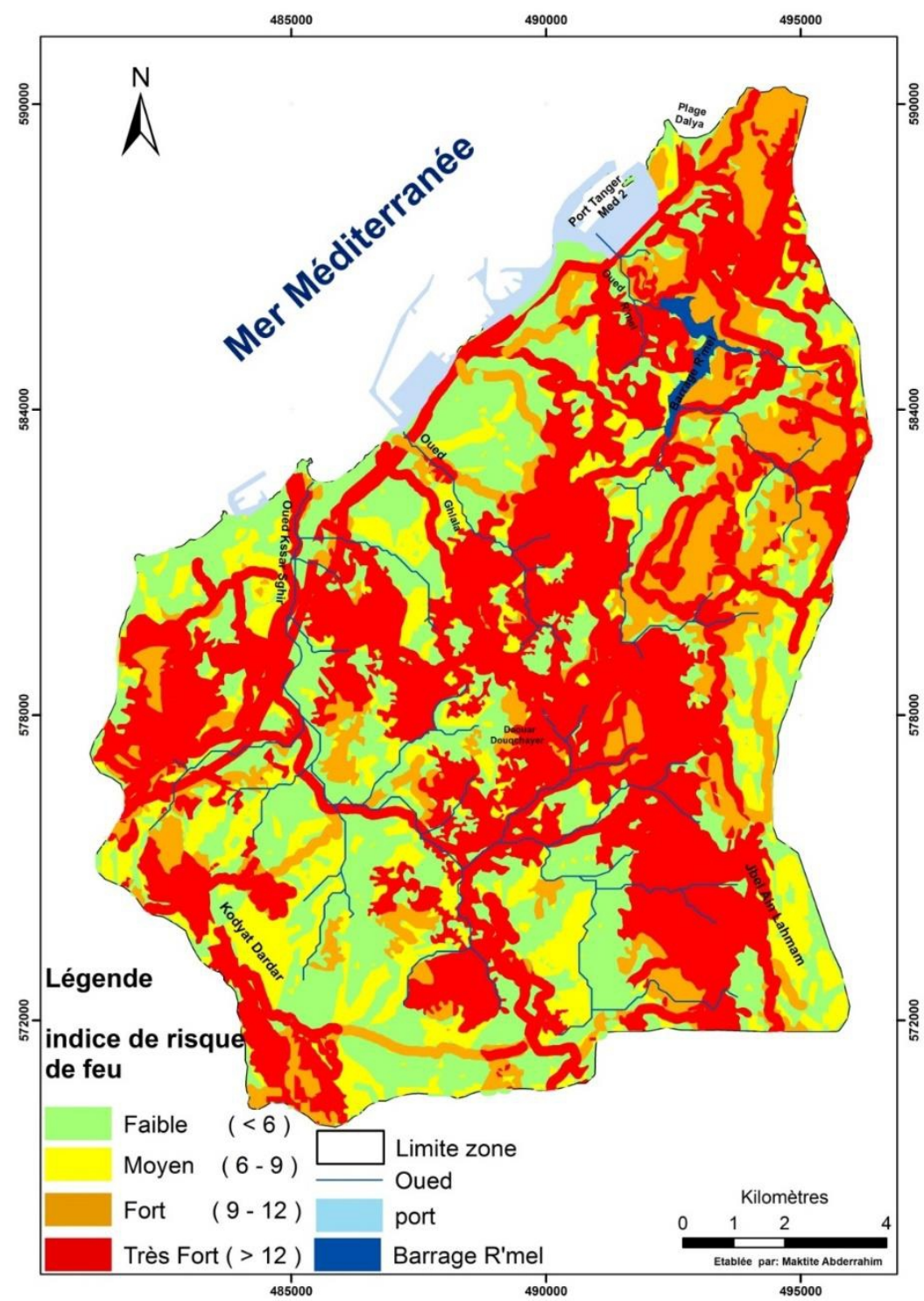

Figure 12. Carte d'indice de risque de feu 
La plupart des zones se trouvent dans un indice de risque moyen et très fort, ces deux classes occupent le Sud-Est, le Nord-Est et toute la partie ouest de la carte. Pour les autres indices, on les trouve au nord et au sud.

\section{Validation du Modèle}

Pour valider ce modèle, une comparaison a été faite entre la carte synthétique de risque de feu et les départs de feu enregistrés en 2015 élaborés par la direction des eaux et forêts de Tanger, cette comparaison permet de prouver les résultats de l'analyse et fournir un élément d'évaluation sur les limites de l'indice IR à partir l'archive des incendies.

La carte de valorisation du modèle (Figure 14) illustre la superposition des points d'incendie et les zones à risque très fort sur la zone d'étude afin de corroborer la carte IR.

Parmi les dix points d'incendies localisés sur la zone d'étude cinq se trouvent sur les zones à risque très fort, quatre sur les zones à risque fort et un seul sur les zones de faible risque (Tableau 10):

Tableau 9. Points d'incendies en 2015

\begin{tabular}{|c|c|c|c|c|c|}
\hline Risque de feu & Très Fort & Fort & Moyen & Faible & $\begin{array}{c}\text { Total } \\
\text { des } \\
\text { points }\end{array}$ \\
\hline Nombre de points & $\mathbf{5}$ & $\mathbf{4}$ & $\mathbf{0}$ & $\mathbf{1}$ & $\mathbf{1 0}$ \\
\hline
\end{tabular}

Les régions affectées par l'incendie pendant l'année de 2015 sont situées dans la partie nord de la zone d'étude, ces zones sont connues par une pression humaine, là où il y a une série d'infrastructures routières (autoroute, route nationale 6 , chemin de fer). 


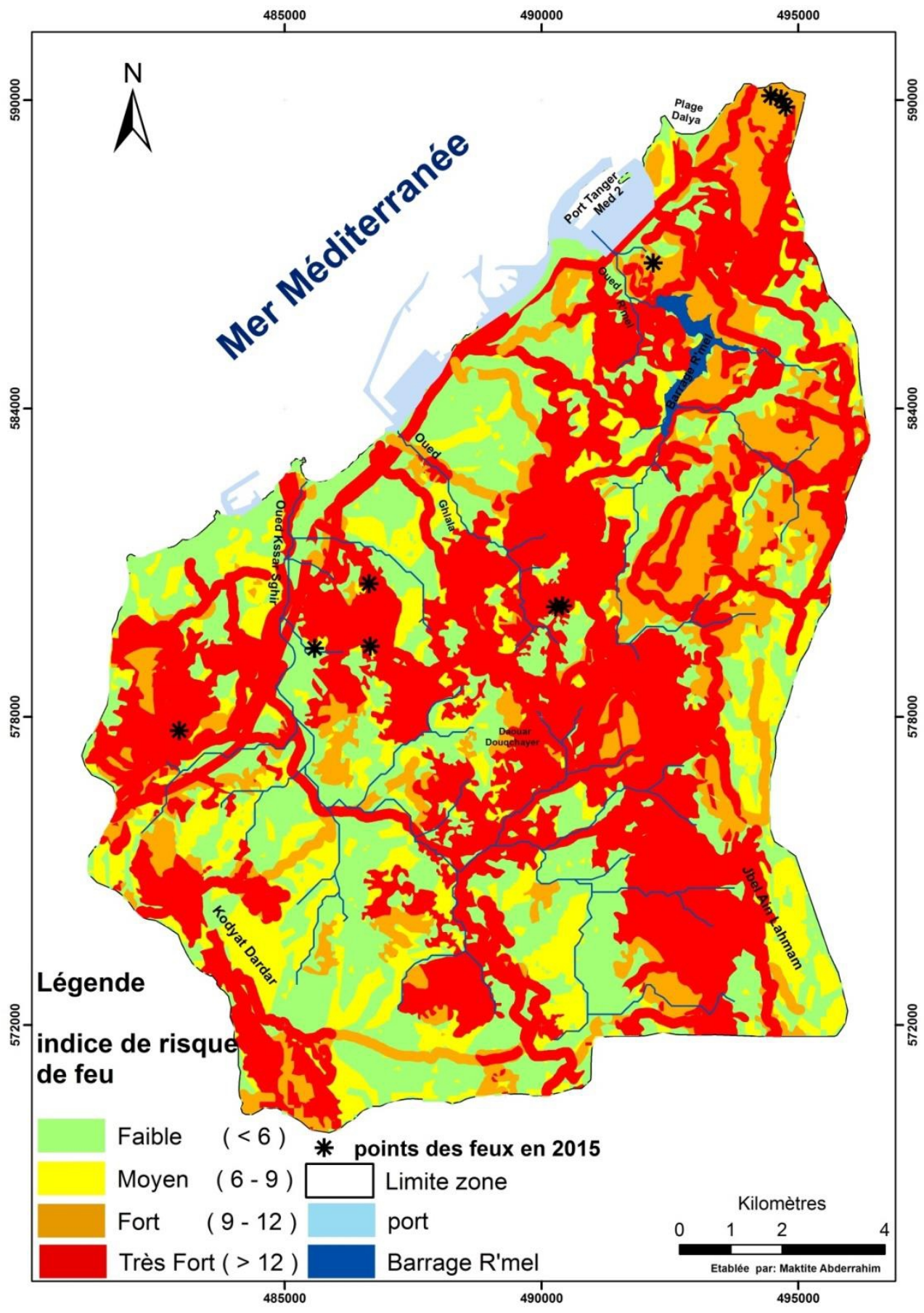

Figure 13. Carte de validation 


\section{Conclusion Générale}

$\mathrm{Au}$ terme de ce travail qui a pour objectif de hiérarchiser la zone d'étude en parcelle en fonction du degré de risque en feu, on a constaté quelques conclusions et résultats préliminaires:

Les incendies des forêts sont dus non seulement à certains effets naturels, mais aussi aux différentes interventions de l'homme, en fonction de l'ampleur et de l'action des groupements humains sur ce milieu, notamment au bord de différentes routes.

Le patrimoine forestier dans la zone d'étude est fortement touché par le phénomène d'incendie, sa régression s'est remarquablement accélérée sous la pression de la démographie.

La carte d'indice topomorphologique fournit des informations sur le risque d'inflammation en se basant sur le degré d'influence de plusieurs facteurs (pente, exposition du versant et l'altitude). Cette carte fait ressortir 4 classes, 61\% des terrains représentent les peu faible à feu, 31\% pour les moyens puis $8 \%$ et $1 \%$ pour les favorables et les très favorables (respectivement).

La carte d'indice de combustibilité permet d'identifier l'interaction d'une espèce avec le feu. La sensibilité de la couverture végétale à l'indice de forêt est fortement liée aux conditions climatiques.

La carte d'indice IC est présentée par quarte classes de la faible à la très élevée, on note la dominante de la classe faible avec $57 \%$ des terrains, suivie par la classe à risque élevé $(22 \%)$, pour la classe modérée et la très élevée, elles présentent $14 \%$ et $6 \%$ respectivement.

Pour la carte d'indice humaine qui représente la superposition de la carte du réseau routier et la carte des zones d'habitat avec la carte des zones forestières, a identifié l'indice de l'impact humain, mis en évidence par le nombre de départ des feux. Cette carte montre l'influence de l'effet humain sur la forêt.

La carte finale qui représente l'indice de risque de feu est définie comme la somme de plusieurs paramètres (l'indice topomorphologie, l'indice de combustibilité et l'indice humain). Les résultats montrent qu'une grande partie de la zone à risque très fort et moyennement fort est due à l'intersection de plusieurs facteurs.

La carte du risque de feu fournie par cette méthode ne permettra pas de lutter contre le feu, mais elle reste un moyen crucial pour informer sur les zones vulnérables aux incendies, afin d'intervenir sur le milieu avec une gestion durable.

La validation du travail par les points des incendies survenus au cours de l'année 2015 enregistrés par l'administration des eaux et forêts, ont prouvé l'efficacité de la méthode sur la zone méditerranéenne malgré l'absence des facteurs anthropiques dans son déroulement. 


\section{References:}

1. Bayo, I. (2016). Que fait le Maroc pour lutter contre les feux de forêts?, Article, Revue electronic The Huffington post Maroc.

2. CEMAGREF (1989). Guide technique du forestier méditerranéen Français, protection des forêts contre les incendies, volume \|\|, France, Fiches 5 et 8 .

3. Dagorne, Y., Duche et al. (1994). Protection des forêts contre les incendies\& Système d'information géologique: Application à la commune d'Auribeau sur Siagne (Alpes Maritimes). Revue forêt méditerranéenne. T: XV, n 4, Octobre 1994, pp. 416-419.

4. DPEFLCD (2015). Bilan des réalisations des actions de prévention et de lutte contre les incendies de forêts, Situation de 09/12/2015, p 1, 2 et 3 .

5. Durand Delga, M., Hottinger, L., Marcais, J., Mattaur, M., Milliard, Y. \& Sauter, G. (1960-1962). Données nouvelles sur la structure de Rif. Livre mémoire. Prof. P. FALLOT, Mémoire hydrologie. France 399-422.

6. Faleh, A., Lakhouja, H., \& Sadiki, A. (2012). SIG, télédétection et évaluation de risque d'incendie de forêts : exemple du massif forestier KHEZANA (province de Chefchaouen), papels de geografia, pp 3756.

7. Guettouche et al. (2010). Modélisation du risque d'incendie de forêts et Validation par Approche Géomatique. Application sur la Forêt du Sahal Algérois, (Algérie), Article, Congrés International GéoTunis. 29 Novembre - 3Décembre 2010.

8. HCEFLCD (2009). Rapport annuel sur les incendies de forêts, Division de la protection des forêts, Rabat, Maroc, 20pp.

9. Mariel, A. (1995). Cartographie du niveau de risque d'incendie: exemple du massif des Maures, CEMAGREF, article.

10. Michard, A. (1976). Elément de géologie Marocaine du Maroc/ Rabat n: $252 / 420$ p.

11. Saidi, A., Missoumi, A., \& Chercheur en géomatique. Forest Fire prediction. The GIS Simulation Model, article

12. TMSA (2008). Projet d'extension du complexe portuaire, (Tanger Med II), $\mathrm{p}: 43$. 